\title{
The effect of mesomorphology upon the
}

\section{performance of nanoparticulate organic photovoltaic}

\section{devices}

Henrik F. Dam ${ }^{a}$, Natalie P. Holmes ${ }^{b}$, Thomas R. Andersen ${ }^{a}$, Thue T. Larsen-Olsen ${ }^{a}$, Matthew

Barr $^{b}$, A. L. David Kilcoyne ${ }^{c}$, Xiaojing Zhou ${ }^{b}$, Paul C. Dastoor ${ }^{b}$, Frederik C. Krebs $^{a}$ and

Warwick J. Belcher ${ }^{b^{*}}$

${ }^{a}$ Department of Energy Conversion and Storage, Technical University of Denmark, Frederiksborgvej 399, DK-4000 Roskilde, Denmark

${ }^{\mathrm{b}}$ Centre for Organic Electronics, University of Newcastle, University Drive, Callaghan NSW 2308, Australia,

${ }^{c}$ Advanced Light Source, Lawrence Berkeley National Laboratory, Berkeley, CA94720, USA

* Corresponding author. Tel: +61 24921 6360, Fax: +61 24921 6907, E-mail address:

Warwick.Belcher@newcastle.edu.au

\begin{abstract}
Scanning transmission X-ray microscopy (STXM) compositional mapping has been used to probe the mesomorphology of nanoparticles (NPs) synthesized from two very different polymer:fullerene blends: poly(3-hexylthiophene) (P3HT) : phenyl-C61-butyric acid methyl ester (PCBM) and poly[4,8-bis(2-ethylhexyloxy)benzo(1,2-b:4,5-b')dithiophene-alt-5,6-
\end{abstract}


bis(octyloxy)-4,7-di(thiophen-2-yl)(2,1,3-benzothiadiazole)-5,5'-diyl] (PSBTBT) : PCBM. The STXM data shows that both blends form core-shell NP structures with similar shell compositions, but with different polymer:fullerene ratios in the core regions. P3HT:PCBM and PSBTBT:PCBM NP organic photovoltaic (OPV) devices have been fabricated and exhibit similar device efficiencies, despite the PSBTBT being a much higher performing low band gap material. By comparing the measured NP shell and core compositions with the optimized bulk hetero-junction (BHJ) compositions, we show that the relatively higher performance of the P3HT:PCBM NP device arises from the fact that its shell composition is much closer to the optimal BHJ value than that of the PSBTBT:PCBM NP device.

KEYWORDS: Nanoparticle, morphology, organic photovoltaic

\section{Introduction}

The incorporation of nanoparticle (NP) organic thin films into organoelectronic devices is a rapidly developing area of research as they offer significant potential advantages over conventionally processed organic thin films. The materials may be processed from aqueous dispersions eliminating the safety and cost issues associated with large scale processing from organic solvents[1,2]. More importantly, the nanoparticulate structure offers the opportunity to control the semi-conducting polymer and blend morphology on the nanoscale[3,4]. Consequently, NP organic thin films are increasingly finding application in areas such as thin film transistors[5], light-emitting diodes[6], and, in particular, photovoltaics[7,8] where roll to roll printing of NP OPV devices has been demonstrated $[9,10]$ and power conversion efficiencies (PCEs) of up to $2.5 \%$ have already been achieved for small area devices[11]. 
Over the past few years, advanced microscopic techniques have revealed that conventional bulk-heterojunction (BHJ) active layers are structured on a hierarchy of length scales; ranging from atomic level interactions between component molecules/polymers (sub-nm) to the formation of ordered domains (a few tens of nm) to surface energy driven large scale phase segregation (up to microns)[12]. The precise nature of the material interactions on all of these length scales is critical to device function; if any of them are detrimental device performance suffers. In BHJ devices, the ordering on all of these length scales is driven by the material thermodynamic properties and can only be indirectly influenced by experimental levers[13]. Consequently, optimisation of the device structure occurs primarily via trial and error and is empirically determined for each material system.
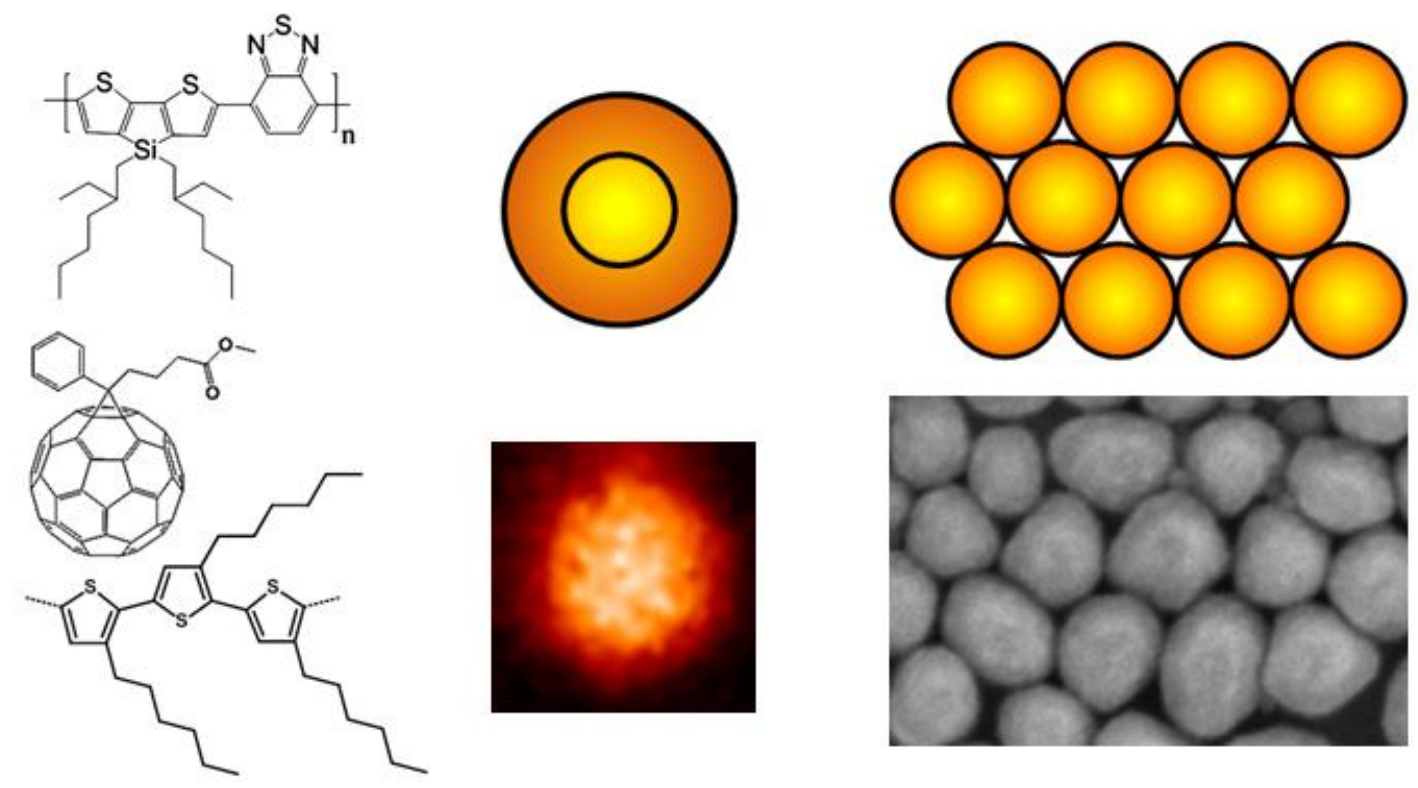

Figure 1: A schematic showing the three length scales on which material interactions occur within the NP approach; (a) the nanoscale $(1-10 \mathrm{~nm}$, demonstrated by skeletal formulae of PSBTBT, PCBM and P3HT from top to bottom), (b) the mesoscale (10 - $100 \mathrm{~nm}$ demonstrated 
by the core-shell nanoparticle structure of a 1:1 P3HT:PCBM nanoparticle observed via scanning transmission X-ray microscopy (STXM)), and (c) the macroscale (> $100 \mathrm{~nm}$, demonstrated by the hexagonal close-packing in 1:1 $\mathrm{P} 3 \mathrm{HT}: \mathrm{PCBM}$ nanoparticles observed via transmission electron microscopy (TEM)).

Uniquely, the NP approach confines device structure on three length scales: (a) molecular/polymer interactions on the nanometer scale (the nanoscale), (b) internal structure of the NP on the scale of a few tens of nanometers (the mesoscale) and (c) the arrangement of NPs in the active layer film (the macroscale) (Figure 1).

Researchers have begun to understand the structural motif of the nanoparticles themselves[3,4]; revealing that the NPs typically have core-shell structures where the core and shell differ in blend composition[3]. However, little work has been undertaken to determine how the nature of the semiconducting materials determines this mesomorphology and how it then influences the performance of the NP OPV devices.

In this paper, we investigate the mesomorphology and device performance of NP OPV devices for two very different polymer:fullerene blends; poly(3-hexylthiophene) (P3HT) : phenyl-C61butyric acid methyl ester (PCBM) and poly[4,8-bis(2-ethylhexyloxy)benzo(1,2-b:4,5b')dithiophene-alt-5,6-bis(octyloxy)-4,7-di(thiophen-2-yl)(2,1,3-benzothiadiazole)-5,5'-diyl]

(PSBTBT) : PCBM. These polymer:fullerene blends have been shown to produce BHJ OPV devices with PCEs in the $3-5 \%$ and $4-6 \%$ range, respectively, and with an active layer phase segregation which is near optimal for charge generation. Here we show that the materials dictate the mesomorphology of the NPs. Using scanning transmission X-ray microscopy (STXM), we show how the composition of the core and shell regions dictates the performance of the NP 
devices, even though the structures are very similar for the two blend systems. In particular, we demonstrate that controlling the composition of the shell is critical in realising the full potential of a given material system.

\section{Results and discussion}

In order to investigate and understand the effects that the active layer components have upon NP mesomorphology, NPs were fabricated from two different polymer:fullerene blends. A 1:1 P3HT:PCBM blend was chosen as the first material system since it is the most studied system in the literature[14], with the material properties of the components and blend, and the physical properties and performance of the resulting OPV devices well established[14].

A 1:1 PSBTBT:PCBM blend was chosen as the second material system since it is representative of the recently developed low band gap polymers for OPV devices[15,16], and has shown promise in tandem OPV devices[17,18]. Whereas P3HT is a largely amorphous polymer in the as-spun state and must be thermally treated to produce a more crystalline material[19,20], in contrast PSBTBT is a highly crystalline polymer[15,21-23]. Upon blending with a fullerene, PSBTBT:fullerene blends display excellent charge transport properties, with little recombination occurring[23].

Static water contact angles for P3HT $\left(106 \pm 0.3^{\circ}\right)[24]$, PSBTBT $\left(104.3^{\circ}\right)[23]$ and PCBM $\left(50.6^{\circ}\right)[23]$ have been reported. While contact angles for conducting polymers are highly dependent upon the level of doping of the polymer sample, these values indicate that the surface energy of PSBTBT is similar to that of P3HT. Consequently, PSBTBT would be expected to form core-shell NPs in a PCBM blend in the same way that P3HT does, with a polymer-rich shell and a fullerene-rich core. 

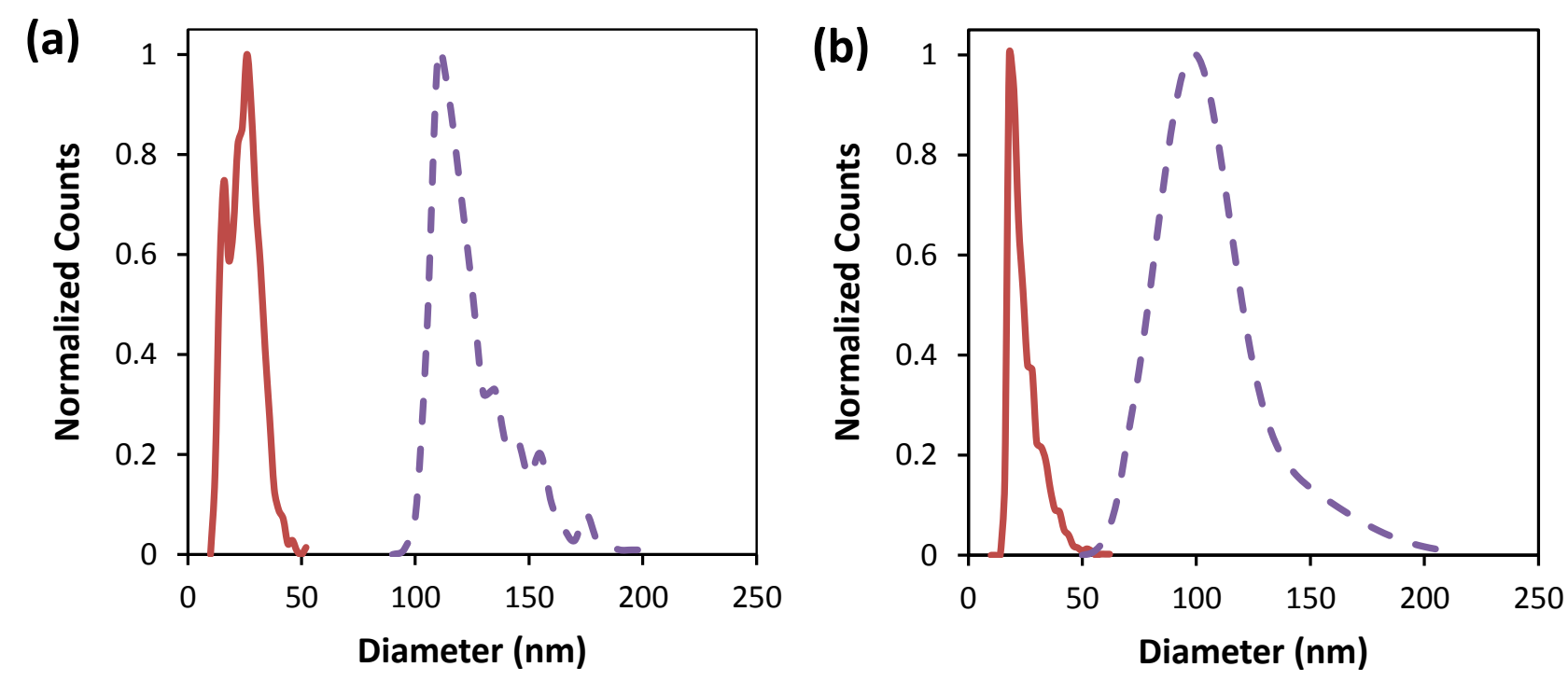

Figure 2: Particle size distributions as detected in reference TEM images of the P3HT:PCBM (a) and PSBTBT:PCBM (b) 1:1 blend particles used for device fabrication (red solid lines) and for STXM measurements (purple dashed lines).

Nanoparticles of 1:1 blends of both P3HT:PCBM and PSBTBT:PCBM were prepared using the standard miniemulsion method reported in the literature[7]. As discussed elsewhere[25], particles of two size ranges were prepared for device characterization and scanning transmission X-ray microscopy (STXM) measurements by varying the amount of surfactant added to the emulsion. Figure 2 shows plots of the size of these particles as extracted from TEM images by a circular Hough particle screening algorithm. For P3HT:PCBM mean particle diameters of 23.8 $\pm 7 \mathrm{~nm}$ and $127 \pm 29 \mathrm{~nm}$ are observed for the small and large particles, respectively, and for PSBTBT:PCBM particles, mean particle diameters of $23.5 \pm 7 \mathrm{~nm}$ and $104 \pm 34 \mathrm{~nm}$, are observed for small and large particles. The error refers to the standard deviation of the particle distribution. 

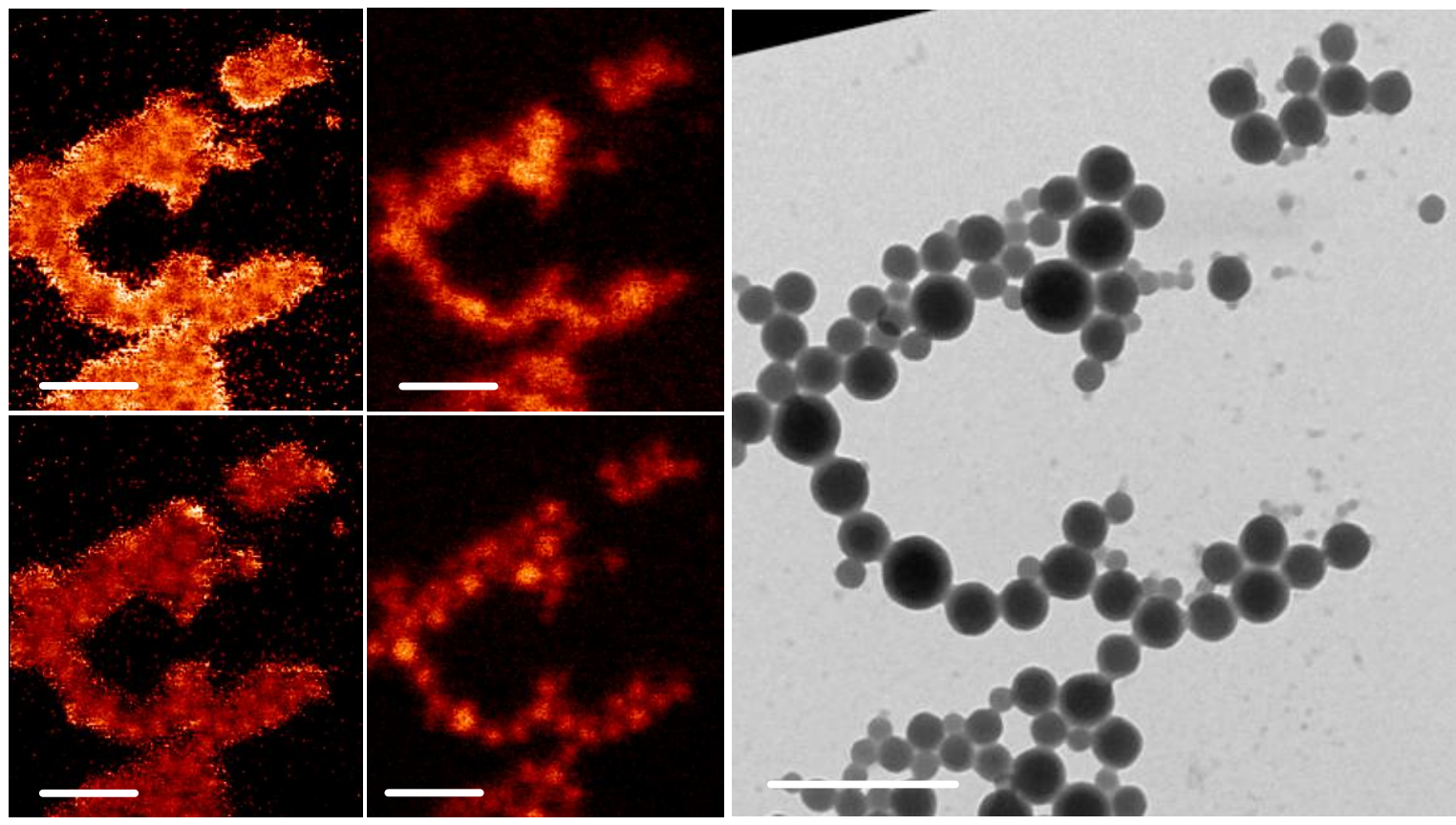

Figure 3: STXM maps showing the fractional composition of (a) PSBTBT and (b) PCBM with corresponding STXM mass plots (c and d) for unannealed PSBTBT:PCBM NPs; (e) matching TEM. In the STXM composition images lighter colours indicate greater fractional composition. All scale bars are $500 \mathrm{~nm}$. 


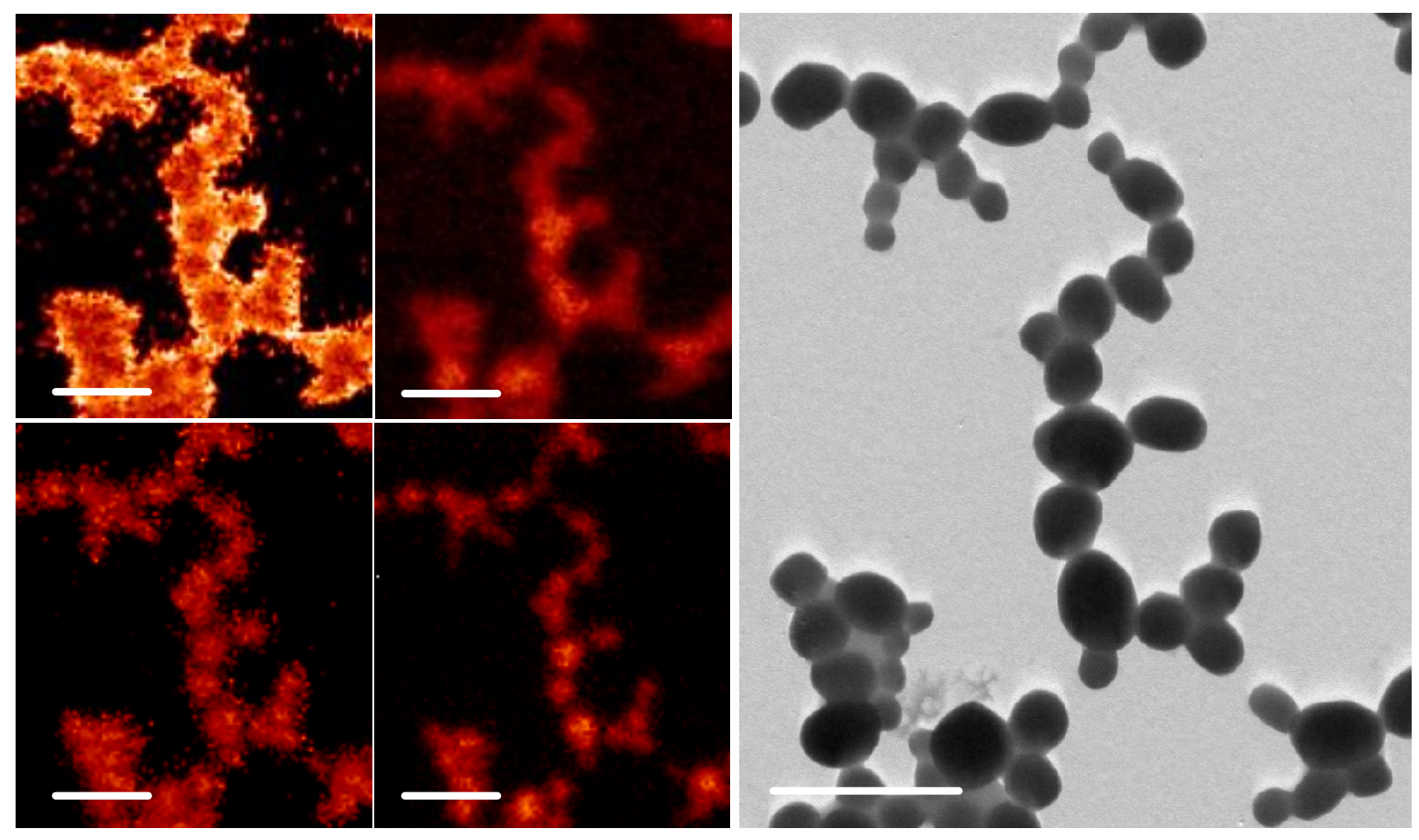

Figure 4. STXM maps showing the fractional composition of (a) P3HT and (b) PCBM with corresponding STXM mass plots (c and d) for unannealed P3HT:PCBM NPs; (e) matching TEM. In the STXM composition images lighter colours indicate greater fractional composition. All scale bars are $500 \mathrm{~nm}$.

Figures 3 and 4 show transmission electron microscopy (TEM) and STXM images for both the large PSBTBT:PCBM and P3HT:PCBM particles, respectively. The TEM images confirm that both sets of materials produce discrete particles in a range of sizes. These images further show that the PSBTBT:PCBM particles are spherical (with aspect ratios close to ideal at 1:0.97 \pm 0.02 for 10 particles), whilst the P3HT:PCBM particles are slightly ovoid in nature with an average aspect ratio (10 particles) of 1:0.91 \pm 0.07 . The STXM images show that all of the NPs for both sets of materials are, as expected, core shell in nature with a polymer-rich shell and a fullerenerich core and that this core-shell mesomorphology occurs at all scale levels down to the resolution limit of the STXM technique $(25 \mathrm{~nm})[3]$. 

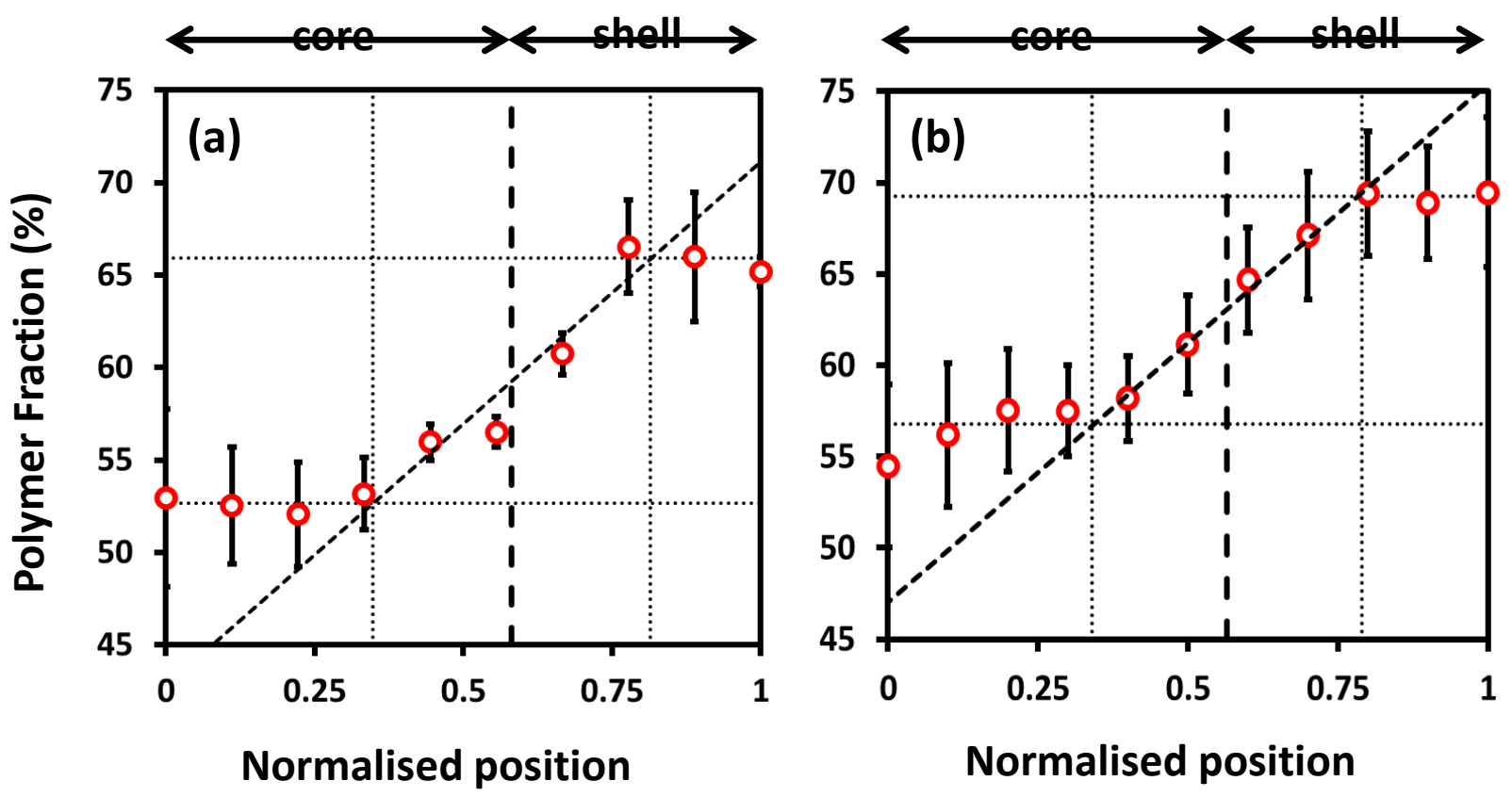

Figure 5: Radially averaged compositional particle cross-sections for an average of five P3HT:PCBM (a) and PSBTBT:PCBM (b) NPs imaged by STXM. The data is presented in the form of a plot of polymer fraction versus a normalized position in the particle. The error bars represent the standard deviations of the compositional data. The calculated interface between the core and shell regions of the particles is shown by the vertical, heavy dashed line.

Figure 5 shows the results of radial compositional averaging of a number of representative particles from both the P3HT:PCBM and PSBTBT:PCBM STXM images. The plots show the average polymer fraction for five particles as a function of distance from the centre of the particle, where the $\mathrm{x}$-axis has been normalized to particle radius. The $\mathrm{x}$-axis origin then represents the polymer fraction at the core of the particle, whilst the $\mathrm{x}$-axis terminus is the polymer fraction at the outer shell. The standard deviation of the measured compositions is presented as error bars on the plots. Note that because STXM is a transmission technique determination of the actual core composition requires deconvolution of the overlying and underlying polymer-rich shell regions. Consequently, the values for polymer fraction within the 
core region shown in the plots in Figure 5 do not reflect the actual core values but rather the summation of the vertical axis through the whole particle. For the shell region the composition is measured directly from the composition maps.

The location of the interfaces between the core and shell regions has been determined as follows. Both data sets show a sigmoidal form, which we approximate as three linear regions. Loci of constant polymer fraction are observed in both the inner core and outer shell regions of the particles connected by an interfacial region of increasing polymer fraction. The dotted horizontal lines define the upper and lower bounds of average polymer fraction and define the inner core and outer shell regions for both sets of particles. The fine dashed line denotes the interfacial region. The interface (heavy dashed line) is then defined as the point that bisects the intercepts of the interfacial region with the lower and upper bounds.

Table 1: Calculated interface position, core volume and composition, shell volume and composition for 1:1 P3HT:PCBM and 1:1 PSBTBT:PCBM NPs. The average error in fractional composition for the interface data points was used to create upper and lower bound lines for the linear interfacial region. The intersection of these bound lines with the core fractional composition was used to define the approximate error in the interface position. This error in the interface position was then used to calculate the approximate errors in the core and shell volumes.

\begin{tabular}{|c|c|c|c|c|c|}
\hline NP system & $\begin{array}{c}\text { Interface } \\
\text { Position }(\% \\
\text { radius })\end{array}$ & $\begin{array}{c}\text { Core } \\
\text { Composition } \\
(\% \text { PCBM })\end{array}$ & $\begin{array}{c}\text { Core Volume } \\
(\% \text { volume })\end{array}$ & $\begin{array}{c}\text { Shell } \\
\text { Composition } \\
(\% \text { polymer })\end{array}$ & $\begin{array}{c}\text { Shell Volume } \\
(\% \text { volume })\end{array}$ \\
\hline P3HT:PCBM & $58.1 \pm 5.2$ & $80 \pm 10$ & $19.6 \pm 5.3$ & $67 \pm 2$ & $80.4 \pm 5.3$ \\
\hline PSBTBT:PCBM & $56.5 \pm 10.4$ & $60 \pm 10$ & $18.0 \pm 10.0$ & $69 \pm 3$ & $82.0 \pm 10.0$ \\
\hline
\end{tabular}


The actual core compositions are determined by taking into account the regions of shell and interface both above and below the core as has been described in detail elsewhere[4]. The shell composition was taken as the average of the locus of constant composition in the outer shell region. Table 1 shows the core and shell compositions for the P3HT:PCBM and PSBTBT:PCBM NPs and reveals that the shell compositions are almost identical. By contrast, the core compositions are very different; the core of the P3HT:PCBM NPs is considerably richer in PCBM relative to that of the PSBTBT:PCBM NPs. The position of the interface between the core and shell is almost identical for both sets of particles and occurs at 0.58 and 0.57 of the particle radius for the P3HT:PCBM and PSBTBT:PCBM NPs respectively. Therefore, the PCBM-rich core region constitutes approximately only $20 \%$ and $18 \%$ by volume of the P3HT:PCBM and PSBTBT:PCBM NPs respectively.

The STXM data shows that all of the imaged nanoparticles for both material systems have the same core-shell mesomorphology at all scale levels down to the resolution limit of the STXM technique $(25 \mathrm{~nm})$ [3]. Consequently, we can apply the parameters in Table 1 to calculate the core and shell dimensions of the small P3HT:PCBM and PSBTBT:PCBM NPs which were synthesized for device fabrication. Figure 6 shows a schematic of the small P3HT:PCBM and PSBTBT:PCBM NPs together with the corresponding compositional data presented now as a polymer:fullerene blend ratio. 


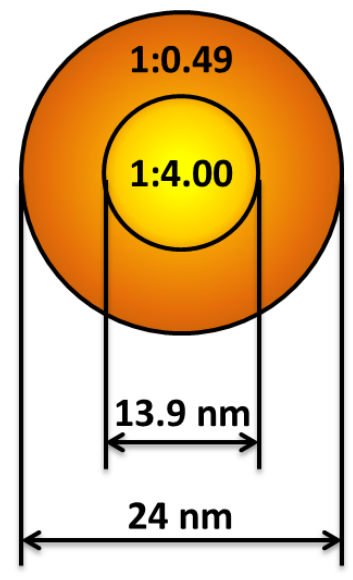

1:1 P3HT:PCBM

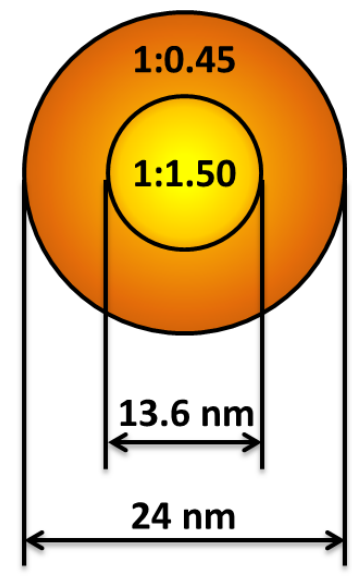

1:1 PSBTBT:PCBM

Figure 6: Schematic of small 1:1 P3HT:PCBM and PSBTBT:PCBM NPs showing core and shell diameters and the polymer:PCBM ratio in the core and shell regions.

A number of structural features of the NPs in Figure 6 are notably similar. Firstly, both the external and core diameters of the particles are identical to within experimental error. Importantly, the core radius of $\sim 7 \mathrm{~nm}$ compares favourably with the exciton diffusion length of $\sim 10 \mathrm{~nm}$ generally accepted for these semiconducting materials[26]. Secondly, the material ratios in the shell regions are also very similar, with approximately twice as much polymer as fullerene present in the blend. By contrast, the core blend ratios for the two sets of NPs are very different, with a much more pure PCBM core present in the P3HT:PCBM particles ( 80\% $\mathrm{PCBM})$ as compared to the PSTBTB:PCBM particles ( $60 \%$ PCBM).

The STXM measurements indicate that while the NP structures are dimensionally optimal (for excitons generated in both shell and core to be harvested), the same is not necessarily true for the blend compositions. For P3HT:PCBM BHJ OPVs the optimal blend ratio is 1:0.8, which corresponds to an optimal polymer fraction of $0.56[27,28]$. By contrast, the optimal composition reported for PSBTBT:PCBM BHJ OPVs is 1:1.5 and corresponds to an optimal polymer fraction 
of 0.40 [23]. Previous studies have shown that the device performance decreases significantly the further the polymer fraction is from its optimal value[29,30].

Comparing the NP core and shell polymer fraction with the optimal values, we note that while the P3HT:PCBM NP shell polymer fraction (0.67) is only $20 \%$ higher than the optimal value (0.56), the PSBTBT:PCBM NP shell polymer fraction (0.69) is over $70 \%$ higher than the optimal value (0.40). For the core regions, the situation is reversed, with the PSBTBT:PCBM NP core polymer fraction at the optimal value whereas the P3HT:PCBM NP core polymer fraction $(0.20)$ is $64 \%$ lower than the optimal value. Consequently, we would expect the shell region to dominate charge generation for the P3HT:PCBM NPs whereas for the PSBTBT:PCBM NPs the core should be optimal. However, given that the core is only $18 \%$ of the total NP volume, and that charges generated in the core still have to traverse the fullerene-depleted shell region, we would expect the performance of the PSBTBT:PCBM NP devices (relative to the optimal BHJ device) to be lower than that for the P3HT:PCBM NP devices, despite the fact that the PSBTBT is the higher performing polymer.

Table 2: Device characteristics for OPV devices fabricated with 1:1 P3HT:PCBM and 1:1 PSBTBT:PCBM NP and BHJ active layers. Characteristics are presented for unannealed NP and BHJ devices and annealed BHJ devices.

\begin{tabular}{|c|c|c|c|c|c|c|c|c|c|c|}
\hline & \multicolumn{5}{|c|}{ P3HT:PCBM } & \multicolumn{5}{|c|}{ PSBTBT:PCBM } \\
\hline Active layer & $\begin{array}{l}\mathrm{V}_{\mathrm{oc}} \\
(\mathrm{V})\end{array}$ & $\begin{array}{c}\mathrm{J}_{\mathrm{sc}} \\
\left(\mathrm{mA} / \mathrm{cm}^{2}\right)\end{array}$ & $\mathrm{FF}$ & $\begin{array}{l}\text { PCE } \\
(\%)\end{array}$ & Ref. & $\begin{array}{l}\mathrm{V}_{\mathrm{oc}} \\
\text { (V) }\end{array}$ & $\begin{array}{c}\mathbf{J}_{\mathrm{sc}} \\
\left(\mathrm{mA} / \mathrm{cm}^{2}\right)\end{array}$ & $\mathrm{FF}$ & $\begin{array}{l}\text { PCE } \\
(\%)\end{array}$ & Ref. \\
\hline $\begin{array}{c}\mathrm{NP} \\
\text { Unannealed } \\
\end{array}$ & 0.52 & 5.5 & 0.47 & 1.3 & [25] & 0.62 & 6.2 & 0.33 & 1.3 & $\begin{array}{c}\text { this } \\
\text { work } \\
\end{array}$ \\
\hline $\begin{array}{c}\text { BHJ } \\
\text { Unannealed }\end{array}$ & 0.61 & 3.8 & 0.38 & 1.0 & [31] & 0.59 & 14.9 & 0.49 & 4.3 & [23] \\
\hline $\begin{array}{c}\text { BHJ } \\
\text { Annealed }\end{array}$ & 0.60 & 9.1 & 0.55 & 3.3 & [31] & 0.60 & 16.4 & 0.53 & 5.2 & [23] \\
\hline
\end{tabular}




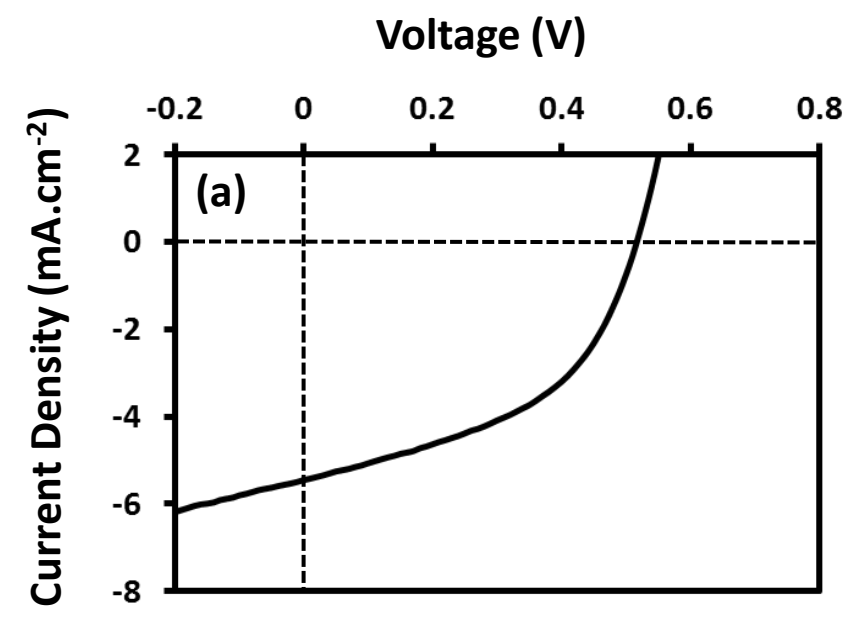

Voltage (V)

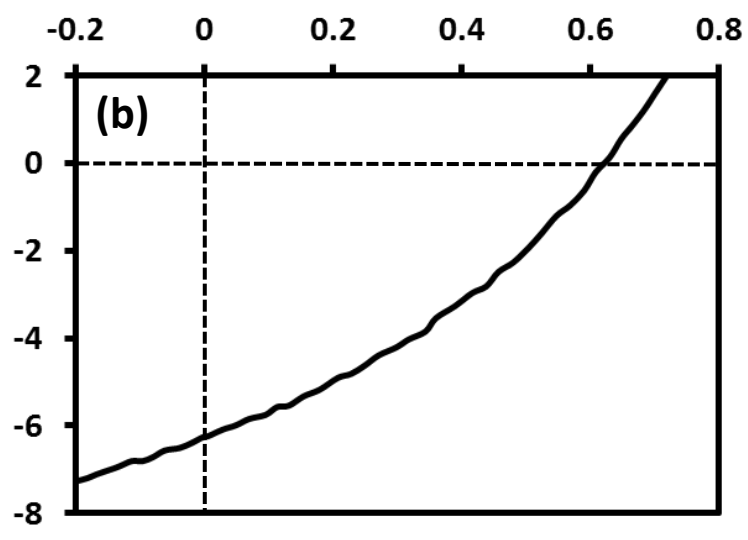

Figure 7: J-V curves for the unannealed 1:1 P3HT:PCBM and PSBTBT:PCBM NP OPV devices presented in Table 2.

Table 2 shows the device characteristics for OPV devices made from the 1:1 P3HT:PCBM and 1:1 PSBTBT:PCBM NPs shown in Fig. 5, together with typical optimised 1:1 P3HT:PCBM and 1:1.5 PSBTBT:PCBM BHJ devices from the literature, processed from chloroform and 1,2dichlorobenzene, respectively. From Table 2 it is clear that P3HT:PCBM BHJ devices improve dramatically upon thermal annealing, as a result of crystallization of the P3HT and ejection of PCBM with a resulting improvement in the overall morphology of the active layer[32]. On the other hand, the PSBTBT:PCBM BHJ devices show a much reduced effect of annealing as a result of the higher initial crystallinity of the PSBTBT polymer[23]. Comparing the two annealed BHJ devices, the PSBTBT performs better than P3HT solely as a result of improved $\mathrm{J}_{\mathrm{sc}}$; consistent with PSBTBT being a low band gap material.

Comparing the NP and BHJ devices, we note that, as predicted, the ratio of the NP:BHJ power conversion efficiency is higher for the P3HT:PCBM NP device (0.39) than for the PSBTBT:PCBM NP device (0.25), despite the fact that the PSBTBT is the higher performing 
polymer in the BHJ blend. Furthermore, the higher NP:BHJ efficiency ratio for P3HT:PCBM is dominated by the improved NP:BHJ $\mathrm{J}_{\mathrm{sc}}$ ratio in the P3HT:PCBM NP device (0.60) compared to the PSBTBT:PCBM NP device (0.38). These results are consistent with the predictions made from the STXM composition measurements and highlight the importance of the role that NP mesomorphology plays in determining device performance.

Based on the STXM and device measurements it is possible to make the following observations. In the P3HT:PCBM NP device charge generation occurs primarily in the shell region since: (1) the composition of this region is closest to the optimal material blend ratio, (2) the shell constitutes $>80 \%$ of the particle volume. Moreover, excitons generated in the core region are also within the accepted diffusion length of a material interface and as such, the entire particle can contribute to charge generation. Indeed, the improvement of the P3HT:PCBM NP device over the unannealed P3HT:PCBM BHJ devices shows that the NP structure can improve device efficiency even though the composition is non-optimal. For the PSBTBT:PCBM device, whereas the core composition is optimal for charge generation, the shell (which still constitutes the bulk of the NP) has a composition that is far from the optimized blend ratio. Consequently charge generation, $\mathrm{J}_{\mathrm{sc}}$ and device efficiency are reduced dramatically.

This work demonstrates that the NP mesomorphology (encompassing both structure and composition) is a critical determinate of NP OPV device function. It is clear that the shell composition dominates charge generation and transport in these devices; this composition, rather than the overall component blend composition must be optimised for high device performance. Since these polymer:fullerene NPs demonstrate a morphology with a fullerene-rich core and fullerene-poor shell for the PSBTBT device, the NP mesomorphology enforces a shell composition that is low in fullerene and far from optimal, consequently the advantages of the NP 
structure are not fully realized. Thus, immediate improvements in NP OPV device performance are achievable by simply tailoring shell composition to be closer to that of their optimized BHJ counterparts. This can be achieved simply by increasing the fullerene loading in the initial component blend during NP fabrication. Work is currently underway to demonstrate this improvement for these material systems.

\section{Conclusions}

STXM compositional mapping has been used to probe the relationship between mesomorphology and performance of P3HT:PCBM and PSBTBT:PCBM NP OPV devices. By comparing the measured NP shell and core compositions with the optimized BHJ compositions, we show that the relatively higher performance of the P3HT:PCBM NP device arises from the fact that its shell composition is much closer to the optimal BHJ value than that of the PSBTBT:PCBM NP device. We conclude that to truly exploit the NP structure, we need to ensure that the composition (and specifically the shell composition) is optimised.

\section{Methods}

\section{Materials}

PSBTBT was prepared as described in the literature [15]. P3HT and PCBM were purchased from Lumtec.

\section{Nanoparticle fabrication}

P3HT:PCBM and PSBTBT:PCBM 1:1 blend NPs were prepared for both STXM measurements and device fabrication by the methods described previously $[7,9,25]$. Particle size measurements were made by applying a circular Hough transform algorithm on TEM images of spin coated particle monolayers on silicon nitride windows. 


\section{STXM acquisition}

Samples were prepared for scanning transmission X-ray microscopy (STXM) by spinning 2.6 $\mu l$ nanoparticle suspension onto low stress silicon nitride windows with silicon dioxide coating (window dimensions $0.25 \times 0.25 \mathrm{~mm}$, window thickness $15 \mathrm{~nm}$, frame $5 \times 5 \mathrm{~mm}$ ) at $3000 \mathrm{rpm}, 1$ min, low acceleration of $112 \mathrm{rpm} / \mathrm{sec}$. Pristine films of PSBTBT, P3HT, PCBM and sodium dodecyl sulfate (SDS) were also prepared for NEXAFS experiments.

STXM imaging was carried out at the Advanced Light Source on Beamline 5.3.2.2 The samples were rastered with respect to the X-ray beam, mounted in the sample chamber which is backfilled with helium (0.33 atm). Near edge X-ray absorption fine structure (NEXAFS) spectra of pristine samples were measured to choose key absorption energies for nanoparticle sample image scans. For PSBTBT:PCBM NP films three STXM scans at energies of $284.5 \mathrm{eV}, 287.4 \mathrm{eV}$ and $288.2 \mathrm{eV}$ were performed. Details of the STXM acquisition process and analysis for P3HT:PCBM NPs are presented in detail elsewhere [3,4].

\section{TEM acquisition}

Transmission electron microscopy (TEM) images of the NPs were acquired after STXM experiments using a Jeol 1200EXII TEM operating at $80 \mathrm{kV}$.

\section{Device preparation and characterization}

The devices were prepared on glass slides pre-patterned with ITO. The slides were cleaned by ultrasonication for $5 \mathrm{~min}$ in water and $5 \mathrm{~min}$ in isopropanol. The glass/ITO slides were then spincoated with an acetone based solution of $\mathrm{ZnO}$ nanoparticles $\left(25 \mathrm{mg} \mathrm{ml}^{-1}\right)$ [33] at $1000 \mathrm{rpm}$ for 10 seconds and dried on a hotplate for $2 \mathrm{~min}$ at $140{ }^{\circ} \mathrm{C}$. The active layer was formed by spincoating of the aqueous PSBTBT:PCBM nanoparticle ink at $500 \mathrm{rpm}$ for $1 \mathrm{~min}$ and subsequently drying the substrate for $2 \mathrm{~min}$ at $140{ }^{\circ} \mathrm{C}$. A layer of PEDOT:PSS (AGFA Orgacon EP-L 5010, 
diluted with isopropanol 2:1 w/w) was spin-coated at $3000 \mathrm{rpm}$ for $1 \mathrm{~min}$, followed by drying for 2 min at $140{ }^{\circ} \mathrm{C}$. The devices were finalized by thermal evaporation of an $\mathrm{Ag}$ electrode in a vacuum chamber at a pressure of $\leq 5 \times 10^{-6}$ mbar, through a shadow mask giving an active area of $\sim 5 \times 5 \mathrm{~mm}$.

Current-voltage characterization was performed using a metal halide lamp calibrated to 1000 $\mathrm{Wm}^{-2} \mathrm{AM} 1.5 \mathrm{G}$ and a Keithley 2400 source-measure unit.

\section{Acknowledgements}

The Danish Strategic Research Council (DSF 2104-07-0022) is gratefully acknowledged for a PhD scholarship (HD). The University of Newcastle and the Australian Renewable Energy Agency (ARENA) are gratefully acknowledged for PhD scholarships (MB, NH). TA and TL acknowledges support from the Danish National Research Foundation and the National Natural Science Foundation of China (Grant no. 51011130028). The TEM images were collected using the Electron Microscope and X-Ray Unit at the University of Newcastle. This work was performed in part at the Materials node of the Australian National Fabrication Facility. This work has been supported by the US Department of Energy (DE-FG02-98ER45737). The ALS is a national user facility supported by the U.S. Department of Energy under Contract No. DEAC02-05CH11231.

\section{Author Contributions}

The manuscript was written through contributions of all authors. All authors have given approval to the final version of the manuscript. 


\begin{abstract}
Abbreviations
OPV, organic photovoltaic; PCE, power conversion efficiency;; NP, nanoparticle; P3HT, poly(3hexylthiophene); PSBTBT, poly[4,8-bis(2-ethylhexyloxy)benzo(1,2-b:4,5-b')dithiophene-alt-5,6bis(octyloxy)-4,7-di(thiophen-2-yl)(2,1,3-benzothiadiazole)-5,5'-diyl]; PCBM， phenyl C61 butyric acid methyl ester; SDS' sodium dodecyl sulfate; ITO, indium tin oxide; STXM, scanning transmission X-ray microscopy; TEM, transmission electron microscopy; SEM, scanning electron microscopy; DLS, dynamic light scattering; BHJ, bulk heterojunction.
\end{abstract}

\title{
References
}

[1] A.M. Ruder, Potential Health Effects of Occupational Chlorinated Solvent Exposure, Ann. N. Y. Acad. Sci. 1076 (2006) 207-227. doi:10.1196/annals.1371.050.

[2] E.M. Ward, P.A. Schulte, K. Straif, N.B. Hopf, J.C. Caldwell, T. Carreon, et al., Research Recommendations for Selected IARC-Classified Agents, Environ. Health Perspect. 118 (2010) 1355-1362. doi:10.1289/ehp.0901828.

[3] K.B. Burke, A.J. Stapleton, B. Vaughan, X. Zhou, A.L.D. Kilcoyne, W.J. Belcher, et al., Scanning transmission $\mathrm{x}$-ray microscopy of polymer nanoparticles: probing morphology on sub-10 nm length scales, Nanotechnology. 22 (2011) 265710. doi:10.1088/0957-4484/22/26/265710.

[4] N.P. Holmes, K.B. Burke, P. Sista, M. Barr, H.D. Magurudeniya, M.C. Stefan, et al., Nano-domain behaviour in P3HT:PCBM nanoparticles, relating material properties to morphological changes, Sol. Energy Mater. Sol. Cells. 117 (2013) 437-445. doi:10.1016/j.solmat.2013.06.003.

[5] D. Darwis, D. Elkington, S. Ulum, G. Bryant, W. Belcher, P. Dastoor, et al., Novel low voltage and solution processable organic thin film transistors based on water dispersed 
polymer semiconductor nanoparticulates, J. Colloid Interface Sci. 401 (2013) 65-69. doi:10.1016/j.jcis.2013.03.052.

[6] G. Mauthner, K. Landfester, A. Köck, H. Brückl, M. Kast, C. Stepper, et al., Inkjet printed surface cell light-emitting devices from a water-based polymer dispersion, Org. Electron. 9 (2008) 164-170. doi:10.1016/j.orgel.2007.10.007.

[7] A. Stapleton, B. Vaughan, B. Xue, E. Sesa, K. Burke, X. Zhou, et al., A multilayered approach to polyfluorene water-based organic photovoltaics, Sol. Energy Mater. Sol. Cells. 102 (2012) 114-124. doi:10.1016/j.solmat.2012.03.016.

[8] T.T. Larsen-Olsen, T.R. Andersen, B. Andreasen, A.P.L. Böttiger, E. Bundgaard, K. Norrman, et al., Roll-to-roll processed polymer tandem solar cells partially processed from water, Sol. Energy Mater. Sol. Cells. 97 (2012) 43-49. doi:10.1016/j.solmat.2011.08.025.

[9] T.R. Andersen, T.T. Larsen-Olsen, B. Andreasen, A.P.L. Böttiger, J.E. Carlé, M. Helgesen, et al., Aqueous Processing of Low-Band-Gap Polymer Solar Cells Using Roll-to-Roll Methods, ACS Nano. 5 (2011) 4188-4196. doi:10.1021/nn200933r.

[10] T.T. Larsen-Olsen, B. Andreasen, T.R. Andersen, A.P.L. Böttiger, E. Bundgaard, K. Norrman, et al., Simultaneous multilayer formation of the polymer solar cell stack using roll-to-roll double slot-die coating from water, Sol. Energy Mater. Sol. Cells. 97 (2012) 22-27. doi:10.1016/j.solmat.2011.08.026.

[11] S. Ulum, N. Holmes, M. Barr, A.L.D. Kilcoyne, B.B. Gong, X. Zhou, et al., The role of miscibility in polymer:fullerene nanoparticulate organic photovoltaic devices, Nano Energy. (n.d.). doi:10.1016/j.nanoen.2013.03.009. 
[12] S.S. Lee, Y.-L. Loo, Structural Complexities in the Active Layers of Organic Electronics, Annu. Rev. Chem. Biomol. Eng. 1 (2010) 59-78. doi:10.1146/annurevchembioeng-073009-100851.

[13] F. Liu, Y. Gu, J.W. Jung, W.H. Jo, T.P. Russell, On the morphology of polymer-based photovoltaics, J. Polym. Sci. Part B Polym. Phys. 50 (2012) 1018-1044. doi:10.1002/polb.23063.

[14] M.T. Dang, L. Hirsch, G. Wantz, P3HT:PCBM, Best Seller in Polymer Photovoltaic Research, Adv. Mater. 23 (2011) 3597-3602. doi:10.1002/adma.201100792.

[15] J. Hou, H.Y. Chen, S. Zhang, G. Li, Y. Yang, Synthesis, characterization, and photovoltaic properties of a low band gap polymer based on silole-containing polythiophenes and 2, 1, 3-benzothiadiazole, J. Am. Chem. Soc. 130 (2008) 1614416145.

[16] E. Bundgaard, F.C. Krebs, Low band gap polymers for organic photovoltaics, Sol. Energy Mater. Sol. Cells. 91 (2007) 954-985. doi:10.1016/j.solmat.2007.01.015.

[17] S. Sista, M.H. Park, Z. Hong, Y. Wu, J. Hou, W.L. Kwan, et al., Highly efficient tandem polymer photovoltaic cells, Adv. Mater. 22 (2009) 380-383.

[18] N. Li, T. Stubhan, D. Baran, J. Min, H. Wang, T. Ameri, et al., Design of the SolutionProcessed Intermediate Layer by Engineering for Inverted Organic Multi junction Solar Cells, Adv. Energy Mater. 3 (2013) 301-307. doi:10.1002/aenm.201200659.

[19] Y. Kim, S.A. Choulis, J. Nelson, D.D.C. Bradley, S. Cook, J.R. Durrant, Device annealing effect in organic solar cells with blends of regioregular poly(3hexylthiophene) and soluble fullerene, Appl. Phys. Lett. 86 (2005) 063502-063502-3. doi:10.1063/1.1861123. 
[20] T. Erb, U. Zhokhavets, G. Gobsch, S. Raleva, B. Stühn, P. Schilinsky, et al., Correlation Between Structural and Optical Properties of Composite Polymer/Fullerene Films for Organic Solar Cells, Adv. Funct. Mater. 15 (2005) 1193-1196. doi:10.1002/adfm.200400521.

[21] M.C. Scharber, M. Koppe, J. Gao, F. Cordella, M.A. Loi, P. Denk, et al., Influence of the Bridging Atom on the Performance of a Low-Bandgap Bulk Heterojunction Solar Cell, Adv. Mater. 22 (2010) 367-370. doi:10.1002/adma.200900529.

[22] M. Morana, H. Azimi, G. Dennler, H.-J. Egelhaaf, M. Scharber, K. Forberich, et al., Nanomorphology and Charge Generation in Bulk Heterojunctions Based on LowBandgap Dithiophene Polymers with Different Bridging Atoms, Adv. Funct. Mater. 20 (2010) 1180-1188. doi:10.1002/adfm.200900931.

[23] H. Lu, B. Akgun, T.P. Russell, Morphological Characterization of a Low-Bandgap Crystalline Polymer:PCBM Bulk Heterojunction Solar Cells, Adv. Energy Mater. 1 (2011) 870-878. doi:10.1002/aenm.201100128.

[24] L. Robinson, J. Isaksson, N.D. Robinson, M. Berggren, Electrochemical control of surface wettability of poly(3-alkylthiophenes), Surf. Sci. 600 (2006) L148-L152. doi:10.1016/j.susc.2006.03.039.

[25] S. Ulum, N. Holmes, D. Darwis, K. Burke, A.L. David Kilcoyne, X. Zhou, et al., Determining the structural motif of P3HT:PCBM nanoparticulate organic photovoltaic devices, Sol. Energy Mater. Sol. Cells. $110 \quad$ (2013) 43-48. doi:10.1016/j.solmat.2012.11.015.

[26] S. Günes, H. Neugebauer, N.S. Sariciftci, Conjugated Polymer-Based Organic Solar Cells, Chem. Rev. 107 (2007) 1324-1338. doi:10.1021/cr050149z. 
[27] W. Ma, C. Yang, X. Gong, K. Lee, A.J. Heeger, Thermally Stable, Efficient Polymer Solar Cells with Nanoscale Control of the Interpenetrating Network Morphology, Adv. Funct. Mater. 15 (2005) 1617-1622. doi:10.1002/adfm.200500211.

[28] M. Reyes-Reyes, K. Kim, D.L. Carroll, High-efficiency photovoltaic devices based on annealed poly(3-hexylthiophene) and 1-(3-methoxycarbonyl)-propyl-1- phenyl(6,6)C[sub 61] blends, Appl. Phys. Lett. 87 (2005) 083506. doi:10.1063/1.2006986.

[29] C. Müller, T.A.M. Ferenczi, M. Campoy-Quiles, J.M. Frost, D.D.C. Bradley, P. Smith, et al., Binary Organic Photovoltaic Blends: A Simple Rationale for Optimum Compositions, Adv. Mater. 20 (2008) 3510-3515. doi:10.1002/adma.200800963.

[30] D. Chirvase, J. Parisi, J.C. Hummelen, V. Dyakonov, Influence of nanomorphology on the photovoltaic action of polymer-fullerene composites, Nanotechnology. 15 (2004) 1317. doi:10.1088/0957-4484/15/9/035.

[31] B. Xue, B. Vaughan, C.-H. Poh, K.B. Burke, L. Thomsen, A. Stapleton, et al., Vertical Stratification and Interfacial Structure in P3HT:PCBM Organic Solar Cells, J. Phys. Chem. C. 114 (2010) 15797-15805. doi:10.1021/jp104695j.

[32] D.R. Kozub, K. Vakhshouri, L.M. Orme, C. Wang, A. Hexemer, E.D. Gomez, Polymer Crystallization of Partially Miscible Polythiophene/Fullerene Mixtures Controls Morphology, Macromolecules. 44 (2011) 5722-5726. doi:10.1021/ma200855r.

[33] O. Hagemann, M. Bjerring, N.C. Nielsen, F.C. Krebs, All solution processed tandem polymer solar cells based on thermocleavable materials, Sol. Energy Mater. Sol. Cells. 92 (2008) 1327-1335. doi:10.1016/j.solmat.2008.05.005. 
GRAPHICAL ABSTRACT

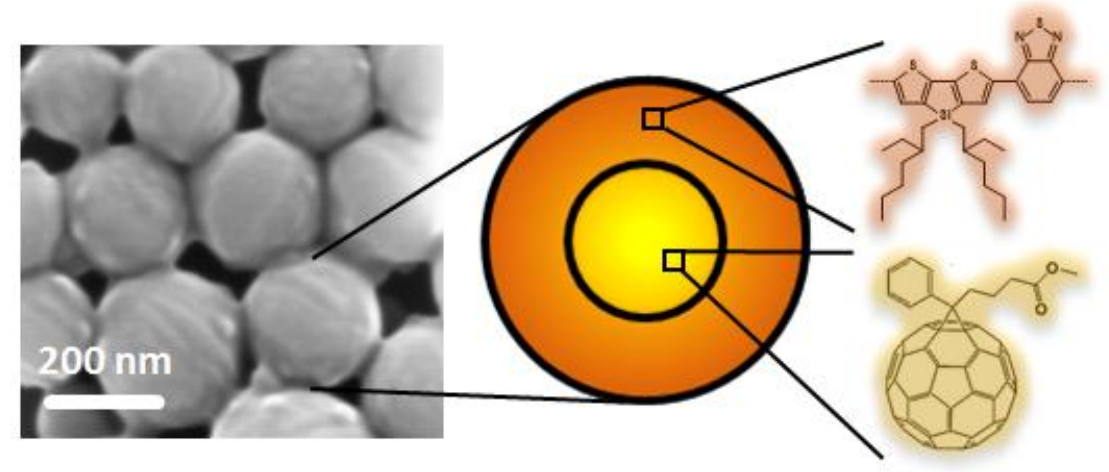

